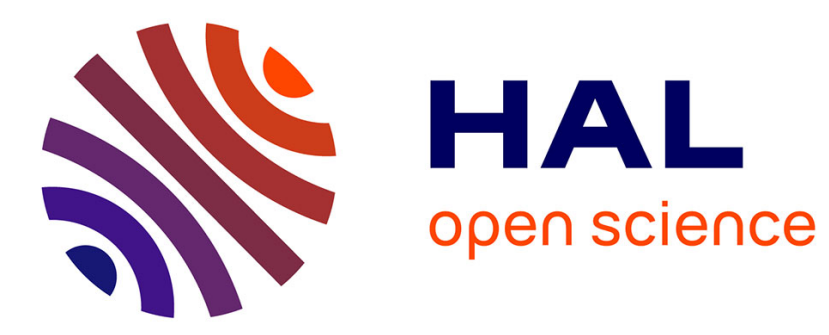

\title{
Appropriate Synthesis of a Crank Rocker Linkage
}

\author{
Joshua K. Pickard, Juan A Carretero
}

\section{To cite this version:}

Joshua K. Pickard, Juan A Carretero. Appropriate Synthesis of a Crank Rocker Linkage. 15th IFToMM World Congress, Jun 2019, Krakow, Poland. hal-02081322

\section{HAL Id: hal-02081322 \\ https://hal.inria.fr/hal-02081322}

Submitted on 27 Mar 2019

HAL is a multi-disciplinary open access archive for the deposit and dissemination of scientific research documents, whether they are published or not. The documents may come from teaching and research institutions in France or abroad, or from public or private research centers.
L'archive ouverte pluridisciplinaire HAL, est destinée au dépôt et à la diffusion de documents scientifiques de niveau recherche, publiés ou non, émanant des établissements d'enseignement et de recherche français ou étrangers, des laboratoires publics ou privés. 


\title{
Appropriate Synthesis of a Crank Rocker Linkage
}

\author{
Joshua K. Pickard ${ }^{1,2}$ and Juan Antonio Carretero ${ }^{2}$ \\ 1 Inria Bordeaux Sud-Ouest, \\ 200 rue vieille tour, 33405 Talence cedex, France \\ Joshua.Pickard@inria.fr \\ 2 University of New Brunswick, \\ 15 Dineen Drive, Fredericton NB, Canada \\ Juan.Carretero@unb.ca
}

\begin{abstract}
The appropriate design methodology is used to model uncertainties and variations in the design of a crank-rocker four-bar linkage. Each design parameter is modelled as a closed interval, such that the appropriate design of a linkage describes a family of linkages. Interval analysis routines are developed and applied to reliably determine the range of performance of the family of linkages. Appropriate synthesis concerns the determination of the complete set of appropriate design solutions which satisfy a given task. Here, a task is modelled with multiple precision points with allowable errors (i.e., boxes). An appropriate design solution must generate a coupler curve which passes within all precision points. Any linkage design contained within the set of appropriate design solutions is guaranteed to satisfy the task requirements.
\end{abstract}

Keywords: Interval analysis, mechanism design, appropriate design, crank-rocker.

\section{Introduction}

The goal of appropriate synthesis is to determine the complete set of design solutions for a mechanism which satisfy some desired task. The appropriate synthesis framework relies on the appropriate design methodology proposed by Merlet and Daney [11]. In this methodology, design parameters are represented as closed intervals, such that an appropriate design describes a family of mechanisms. Uncertainties in the design parameters can be easily accounted for. Interval analysis routines are used to determine the range of performance of the family of mechanisms.

Due to the uncertainties present in an appropriate design, it is not useful to have an exact description of the desired task because the performance cannot be described exactly. Instead, the desired task is described by specifying allowable errors on the various criteria. Then, it is only necessary to check if the criteria are exceeded for a given appropriate design. The performance of a mechanism does not need to be evaluated to a specific resolution. If the worst-case performance of an appropriate design is better than the desired performance, then 
the appropriate design is considered to be a solution to the problem and each mechanism design within the family of mechanisms is also a solution.

Consider the following example. It may be desired to synthesize a fourbar linkage which is able to follow a straight-line trajectory, but rarely is the "straight" criteria strictly necessary. Therefore, an allowable error may be specified such that the goal of the linkage is to approximate the straight-line trajectory without exceeding the allowable error. Appropriate synthesis applied to the four-bar linkage will return every combination of appropriate design parameters which satisfy the desired trajectory.

Useful coupler curves can be generated when the geometric parameters of the linkages are properly selected. The process of selecting these parameters, based on some desired response, is known as dimensional synthesis. The two conventional methods of dimensional synthesis are exact synthesis and approximate synthesis. Exact synthesis of four-bar linkages involves obtaining geometric parameters which produce a coupler curve passing exactly through a set of precision points $[7,12,14]$ or exactly matching a given algebraic coupler curve equation [1]. Approximate synthesis, on the other hand, uses numerical methods to synthesize a linkage that satisfies, typically with minimal error or some other metric, some desired trajectory $[2,3,6]$. Appropriate synthesis of the four-bar linkage requires a different method of dimensional synthesis. This new method allows for the determination of the complete set of design solutions which satisfy the given criteria of the task while also accounting for the variabilities and uncertainties in the design parameters.

The issue of uncertainties in the four-bar linkage plays a significant role in the actual performance of the mechanism. Error in the crank angle results in a translational and rotational error in the coupler point [5]. In the four-bar linkage, uncertainties make it no longer possible to obtain an exact solution for the coupler point and thus exact synthesis methods are not applicable. Instead, the coupler point can only be determined to lie within some bounds, where the bounds are functions of the uncertainties.

In what follows, the uncertainties during synthesis of a crank-rocker mechanism are accounted for, such that the desired performance of a synthesized mechanism is guaranteed. Moreover, the set of appropriate designs which satisfy

the desired criteria is obtained. From this set, the end-user would be free to select the design which best suit their needs. This process is dubbed appropriate synthesis and is described in the rest of this paper.

\section{The Four-bar Linkage}

The four-bar linkage is a planar mechanism consisting of four rigid members: the frame $O_{A} O_{B}$, the input link $O_{A} A$, the output link $O_{B} B$, and the coupler link $\triangle A B C$. These members are connected by four revolute pairs forming a closed-loop kinematic chain of 1-DOF. A point on the coupler $C$, known as the coupler point, traces a path as the input link is rotated. This path is known as the coupler curve. 
a)

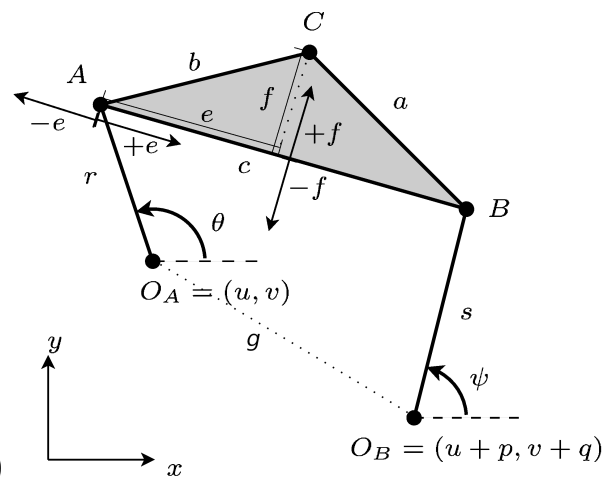

b)

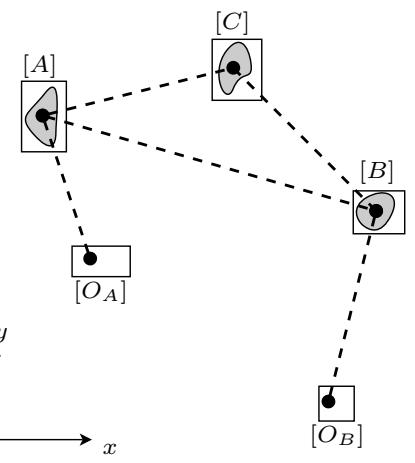

Fig. 1. Four-bar linkage a) basic description and b) effect of geometrical uncertainties.

The four-bar linkage and its associated design parameters are provided in Figure 1a. The fixed base location $O_{A}$ is located at coordinates $(u, v)$ with respect to the reference frame. The location $O_{B}$ is located at coordinates $(p, q)$ with respect to $O_{A}$ or $(u+p, v+q)$ with respect to the reference frame. The input link has an input angle $\theta$ (measured relative to the $\mathrm{x}$ axis) and length $r$. The output link has an output angle $\psi$ (measured relative to the $\mathrm{x}$ axis) and length $s$. The coupler point has the coordinates $C=\left(C_{x}, C_{y}\right)$ and the triangle has edge lengths $a, b$, and $c$. Finally, the parameters $e$ and $f$ are used to describe the location of $C$ relative to the segment $A B(e . g .,+f /-f$ specifies $C$ to the left/right of segment $A B$ ).

Figure $1 \mathrm{~b}$ depicts the effect of uncertainties on the linkage. The fixed locations $O_{A}$ and $O_{B}$ are known to lie in the corresponding boxes $\left[O_{A}\right]$ and $\left[O_{B}\right]$, respectively. For a given input angle $[\theta]$ and output angle $[\psi]$, the locations $A$, $B$, and $C$ will lie in some domain. With interval analysis, these domains are approximated by boxes $[A],[B]$, and $[C]$, respectively. The coupler point will always be inside box $[C]$ for a given input and output angle.

\subsection{Kinematic Equations and Existence Tests}

The equations describing the kinematics of the four-bar linkage can be formulated by solving a set of distance equations $\left(f_{1}\right.$ through $\left.f_{5}\right)$. The vertex $A=\left(A_{x}, A_{y}\right)$ of the coupler triangle lies on the circle centred at $O_{A}$ with radius $r$. The vertex $B=\left(B_{x}, B_{y}\right)$ of the coupler triangle lies on the circle centred at $O_{B}$ with radius $s$. The points $A$ and $B$ are separated by distance $c$. The points $A$ and $B$ are functions of the design parameters and input and output angles $\theta$ and $\psi\left(f_{6}\right.$ through $\left.f_{9}\right)$. The edge lengths $a$ and $b$ of the coupler link are functions of the design parameters $c, e$, and $f\left(f_{10}\right.$ and $\left.f_{11}\right)$. Let $g$ be the distance between points $O_{A}$ and $O_{B}\left(f_{12}\right)$. A unique assembly of the platform is enforced 
by adding constraints $f_{13}$ and $f_{14}$ which take into account the signs of $e$ and $f$.

$$
\begin{aligned}
& f_{1}:=\left\|O_{A} A\right\|^{2}=\left(u-A_{x}\right)^{2}+\left(v-A_{y}\right)^{2}=r^{2} \\
& f_{2}:=\left\|O_{B} B\right\|^{2}=\left((p+u)-B_{x}\right)^{2}+\left((q+v)-B_{y}\right)^{2}=s^{2} \\
& f_{3}:=\|A B\|^{2}=\left(A_{x}-B_{x}\right)^{2}+\left(A_{y}-B_{y}\right)^{2}=c^{2} \\
& f_{4}:=\|A C\|^{2}=\left(A_{x}-C_{x}\right)^{2}+\left(A_{y}-C_{y}\right)^{2}=b^{2} \\
& f_{5}:=\|B C\|^{2}=\left(B_{x}-C_{x}\right)^{2}+\left(B_{y}-C_{y}\right)^{2}=a^{2} \\
& f_{6}:=A_{x}=u+r \cos (\theta) \\
& f_{7}:=A_{y}=v+r \sin (\theta) \\
& f_{8}:=B_{x}=(p+u)+s \cos (\psi) \\
& f_{9}:=B_{y}=(q+v)+s \sin (\psi) \\
& f_{10}:=b=\sqrt{e^{2}+f^{2}} \\
& f_{11}:=a=\sqrt{(c-e)^{2}+f^{2}} \\
& f_{12}:=g=\sqrt{p^{2}+q^{2}} \\
& f_{13}:=C_{x}=A_{x}+1 / c\left(\left(B_{x}-A_{x}\right) e+\left(A_{y}-B_{y}\right) f\right) \\
& f_{14}:=C_{y}=A_{y}+1 / c\left(\left(B_{y}-A_{y}\right) e+\left(A_{x}-B_{x}\right) f\right)
\end{aligned}
$$

Existence tests (e.g., Krawcyzk [9] or Kantorovitch [8]), which will be used to solve two kinematics problems described in Section 4 , can be formulated from Equations $f_{2}-f_{5}$ to give a square system in unknowns $\mathbf{u}=\left(B_{x}, B_{y}, C_{x}, C_{y}\right)$. This allows to determine if there is a unique solution for the unknowns inside some interval $[\mathbf{u}]$. The Jacobian matrix $\mathbf{J}$ and Hessian matrix $\mathbf{H}$ of the corresponding system with these unknowns, which are well formulated for interval analysis, are

$$
\begin{gathered}
\mathbf{J}=\left(\begin{array}{cccccc}
-2 p-2 u+2 B_{x} & -2 q-2 v+2 B_{y} & 0 & 0 \\
-2 A_{x}+2 B_{x} & -2 A_{y}+2 B_{y} & \multicolumn{1}{c}{0} & 0 \\
0 & 0 & -2 A_{x}+2 C_{x}-2 A_{y}+2 C_{y} \\
2 B_{x}-2 C_{x} & 2 B_{y}-2 C_{y} & -2 B_{x}+2 C_{x} & -2 B_{y}+2 C_{y}
\end{array}\right) \\
\mathbf{H}=\left(\begin{array}{cccc|cccc|ccc|ccccc}
2 & 0 & 0 & 0 & 2 & 0 & 0 & 0 & 0 & 0 & 0 & 0 & 2 & 0 & -2 & 0 \\
0 & 2 & 0 & 0 & 0 & 2 & 0 & 0 & 0 & 0 & 0 & 0 & 0 & 2 & 0 & -2 \\
0 & 0 & 0 & 0 & 0 & 0 & 0 & 0 & 0 & 0 & 2 & 0 & -2 & 0 & 2 & 0 \\
0 & 0 & 0 & 0 & 0 & 0 & 0 & 0 & 0 & 0 & 0 & 2 & 0 & -2 & 0 & 2
\end{array}\right)
\end{gathered}
$$

\subsection{Appropriate Design Description}

An appropriate design is described by $[\mathcal{D}]$, whereas an exact design is described by $\mathcal{D}$. The interval domains of the appropriate design parameters account for uncertainties such that:

$$
[\mathcal{D}]=\{[u],[v],[p],[q],[r],[s],[c],[e],[f]\}
$$


The uncertainties on the design parameters may be described by the vector $\Delta \mathcal{D}$ as

$$
\Delta \mathcal{D}=(\Delta u, \Delta v, \Delta p, \Delta q, \Delta r, \Delta s, \Delta c, \Delta e, \Delta f)^{T}
$$

If an exact design $\mathcal{D}$ is considered, then the appropriate design parameters which account for uncertainties will be given by

$$
[\mathcal{D}]=\mathcal{D} \pm \Delta \mathcal{D}
$$

An appropriate synthesis routine will return the complete set of appropriate designs $[\mathcal{D}]$ which satisfy the given task.

\section{Branches and Circuits}

The description for circuits and branches are adopted from [4]. For a given assembly of a four-bar linkage, the coupler point will follow what is referred to as a circuit. To change the circuit being followed, the linkage would need to be disassembled and reassembled. The term toggle position describes when the coupler link $A B$ is collinear with the output link $O_{B} B$. At a toggle position, the linkage is able to change its branch (a branch is defined by a transmission angle, the angle between the coupler and output links, in the range of $(0, \pi)$ or $(-\pi, 0))$.

The number of circuits corresponding to a particular classification of linkages are described in [13]. The ranges of the input and output angles corresponding to a particular classification are provided in [10]. The branch and circuit conditions are extended for appropriate designs as follows. Note that $O_{A} O_{B}$ is used as a reference for the angles in [10]. Here, the angles must be shifted such that for an appropriate design the shifted angles $\left[\theta^{\prime}\right]$ and $\left[\psi^{\prime}\right]$ are

$$
\begin{aligned}
{\left[\theta^{\prime}\right] } & =[\theta]-\operatorname{atan} 2([q],[p]) \\
{\left[\psi^{\prime}\right] } & =[\psi]-\operatorname{atan} 2([q],[p])
\end{aligned}
$$

where atan2 is the quadrant-corrected inverse tangent function.

The limits on the shifted angles $\left[\theta^{\prime}\right]$ and $\left[\psi^{\prime}\right]$ are determined as follows

$$
\begin{aligned}
{\left[\theta_{\text {min }}^{\prime}\right] } & =\operatorname{acos}\left(\left(g^{2}+r^{2}\right)-(c-s)^{2}\right) /(2 r g) \\
{\left[\theta_{\max }^{\prime}\right] } & =\operatorname{acos}\left(\left(g^{2}+r^{2}\right)-(c+s)^{2}\right) /(2 r g) \\
{\left[\psi_{\min }^{\prime}\right] } & =\operatorname{acos}\left((c+r)^{2}-\left(g^{2}+s^{2}\right)\right) /(2 s g) \\
{\left[\psi_{\max }^{\prime}\right] } & =\operatorname{acos}\left((c-r)^{2}-\left(g^{2}+s^{2}\right)\right) /(2 s g)
\end{aligned}
$$

For the crank-rocker linkage, input link can fully rotate and the output link has two operating ranges. The circuits are identified as follows

1. Circuit 1: $\theta^{\prime} \in[0,2 \pi],\left[\psi_{\min }^{\prime}\right] \leq \psi^{\prime} \leq\left[\psi_{\max }^{\prime}\right]$

2. Circuit 2: $\theta^{\prime} \in[0,2 \pi],-\left[\psi_{\max }^{\prime}\right] \leq \psi^{\prime} \leq-\left[\psi_{\min }^{\prime}\right]$ 
To determine the circuit, it is also necessary to know which side of the line $A B$ the coupler point is on. That is, $[f] \geq 0$ corresponds to one circuit, whereas $[f]<0$ corresponds to another circuit.

A given appropriate design $[\mathcal{D}]$ and coupler point $[C]$ may result in multiple assemblies (circuits and branches). It is necessary with appropriate synthesis that certain restrictions be applied to the assemblies. A desired coupler curve can only be achieved with a single circuit. This concept is referred to as an assembly mode defect. An assembly filtering routine is used within the appropriate synthesis method to remove appropriate designs which cannot satisfy the assembly conditions.

\section{Solving the Kinematics}

To solve the kinematics of the four-bar linkage, two main problems are considered:

1. Forward problem: Determine a solution for the coupler point $\left(\left[C_{x}\right],\left[C_{y}\right]\right)$ for a fixed value for $[\theta]$ and an allowable range for $[\psi]$. For this problem, a fixed value for $[\theta]$ is given and $[\psi]$ is given as an allowable range. The goal is to compute the solution/solutions for $\left(\left[C_{x}\right],\left[C_{y}\right]\right)$ and $[\psi]$ which correspond to the fixed value of $[\theta]$.

2. Interior problem: Determine a solution for $[\theta]$ and $[\psi]$ and coupler point $\left(\left[C_{x}\right],\left[C_{y}\right]\right)$ from the interior of the box $([x],[y])$. For this problem, a box $([x],[y])$ is given and $[\theta]$ and $[\psi]$ are given as allowable ranges. The goal is to compute a solution for $[\theta],[\psi]$ and $\left(\left[C_{x}\right],\left[C_{y}\right]\right)$ such that $\left(\left[C_{x}\right],\left[C_{y}\right]\right) \subset$ $([x],[y])$.

The forward problem is useful for the analysis problem of computing the coupler curve associated with an appropriate design. The interior problem is useful for the synthesis problem of determining if an appropriate design satisfies desired coupler curve characteristics, as is required for appropriate synthesis.

For the forward and interior problems, an existence test is able to identify the existence of a unique solution for the coupler point and converge to a box tightly containing the solution. If the existence test succeeds, then a unique solution for the coupler point is found. To create various solving routines, a bisection phase may safely bisect the input and/or output angles $[\theta]$ and $[\psi]$. The bisection of a coordinate may lead to a loss of solutions due to the wrapping effect of interval analysis, whereas the bisection of an angle is safe.

\section{Coupler Curve of an Appropriate Design}

When the design parameters are represented with intervals, identifying the coupler curve becomes a difficult problem. Given an appropriate design $[\mathcal{D}]$ of a linkage, it is possible to represent the coupler curve as a set of boxes which tightly contain the true coupler curves associated with each design $\mathcal{D} \in[\mathcal{D}]$. This provides a description of the worst-case error in the coupler curve. 


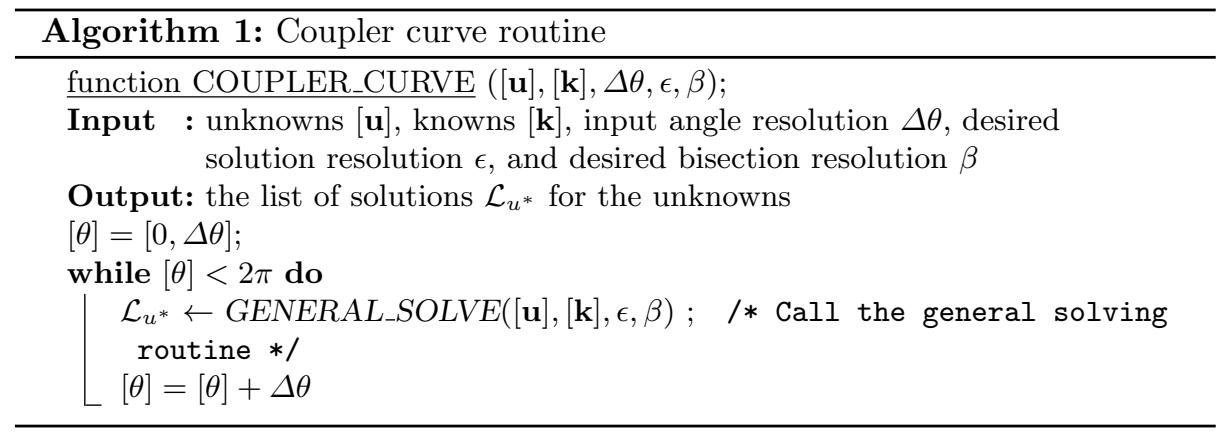

Algorithm 1 solves for the set of coupler point intervals $\left(\left[C_{x}\right],\left[C_{y}\right]\right)$ using the forward problem formulation. GENERAL_SOLVE is a classical branch-andbound routine which applies interval simplification, existence tests, and interval bisection to determine all solutions for the unknowns. The unknowns and knowns here are $[\mathbf{u}]=\left(\left[B_{x}\right],\left[B_{y}\right],\left[C_{x}\right],\left[C_{y}\right]\right)$ and $[\mathbf{k}]=\left([\mathcal{D}],\left[A_{x}\right],\left[A_{y}\right]\right)$. Let $\Delta \theta$ represent the desired width of the input angle interval (the resolution). A loop iterates over all values of $[\theta]$ in the range $[0,2 \pi]$. Inside this loop, the list of corresponding coupler point solutions $\mathcal{L}_{u^{*}}$ are obtained for $[\theta]$.

The coupler curve for a crank-rocker linkage with an appropriate design given by

$$
[\mathcal{D}]=([0],[0],[0.4],[0],[0.1],[0.4],[0.2517],[0.12585],[0.15534])^{T} \pm \Delta \mathcal{D}^{T}
$$

is plotted in Figure 2 for $\Delta \theta=0.001 \mathrm{rad}$. The appropriate design considers an uncertainty of $\Delta \mathcal{D}=0.0001$ added to each design parameter. The desired resolutions are selected as $\epsilon=0.0000001 \mathrm{~m}$ and $\beta=0.0005 \mathrm{rad}$. The Kantorovitch existence method and largest-first bisection are applied here. Each circuit is identified with a different color.

\section{Appropriate Synthesis}

If the set of boxes, pertaining to some portion of the coupler curve are contained inside a desired response, then it is possible to state that the linkage design is guaranteed to satisfy the desired response. In this work, a set of precision points, each with allowable error, are used to describe the desired response (task). That is, a precision point is modelled as $\left(\left[P_{x}\right],\left[P_{y}\right]\right)=\left(P_{x} \pm \Delta P_{x}, P_{y} \pm \Delta P_{y}\right)$, where $\Delta P_{x}$ and $\Delta P_{y}$ are the allowable errors. Appropriate synthesis is used to determine the complete set of design solutions from the design search space, where each design solution must have coupler point solutions that are subsets of the precision points (i.e., $\left.\left(\left[C_{x i}\right],\left[C_{y i}\right]\right) \subset\left(\left[P_{x i}\right],\left[P_{y i}\right]\right)\right)$.

An appropriate synthesis routine is proposed in Algorithm 2, which returns three sets of appropriate design parameters: solutions, boundaries, and nonsolutions for the appropriate designs. A list $\mathcal{L}_{\text {test }}$ contains the set of design 


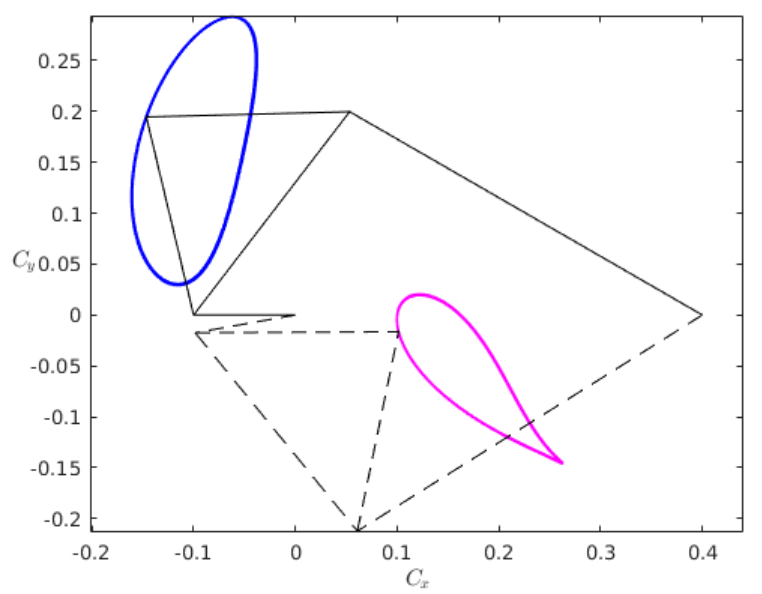

Fig. 2. The coupler curves corresponding to a crank-rocker - equation (9).

parameters being considered. A precision point verification routine is applied to each $[\mathcal{D}]$ in the list $\mathcal{L}_{\text {test }}$, which checks if there is a coupler point solution satisfying each precision point in the desired response $\mathcal{R}$. This routine takes the appropriate design parameters and the input angle resolution $\Delta \theta$ into account when evaluating the coupler point solutions. The resolutions $\epsilon$ and $\beta$ are used as stopping criteria in the existence and bisection methods respectively. Each coupler point solution must correspond to the same circuit. If the routine returns 0 , then bisection is applied to the design parameters $[\mathcal{D}]$; bisection is only applied if width $([\mathcal{D}]) \geq 2 \Delta \mathcal{D}$; otherwise, the design is saved to the boundary set. If the routine returns 1 , then the design is verified and is saved to the solution set. If the routine returns -1 , the design is not a solution and is saved to the non-solution set. The remaining unclassified designs are saved to the boundary set.

A desired response is specified with the following three precision points with full ranges of allowable angles for $[\theta]$ and $[\psi]$ (i.e., $[-\pi, \pi])$ :

$$
\begin{aligned}
& \left(\left[P_{x 1}\right],\left[P_{y 1}\right]\right)=([-0.06,-0.04],[0.15,0.17]) \\
& \left(\left[P_{x 2}\right],\left[P_{y 2}\right]\right)=([-0.14,-0.12],[0.22,0.24]) \\
& \left(\left[P_{x 3}\right],\left[P_{y 3}\right]\right)=([-0.17,-0.15],[0.06,0.08]) .
\end{aligned}
$$

The set of appropriate design solutions and boundaries which yield a crankrocker linkage are plotted in Figure 3. The design parameter search space is defined as $[p] \in[-1.0,1.0]$ and $[q] \in[-1.0,1.0]$. The remaining design parameters are the same as in equation (9). An uncertainty of $\Delta \mathcal{D}=0.001$ is assigned to each design parameter and the input angle uncertainty is set as $\Delta \theta=0.001 \mathrm{rad}$. For the verification routine, the desired resolutions are selected as $\epsilon=0.0000001 \mathrm{~m}$ and $\beta=0.0005 \mathrm{rad}$. The Kantorovitch existence method and largest-first bisection are again applied here. Every value of $p$ and $q$ contained within the appropriate design solutions (green regions) are guaranteed to yield a crank-rocker 


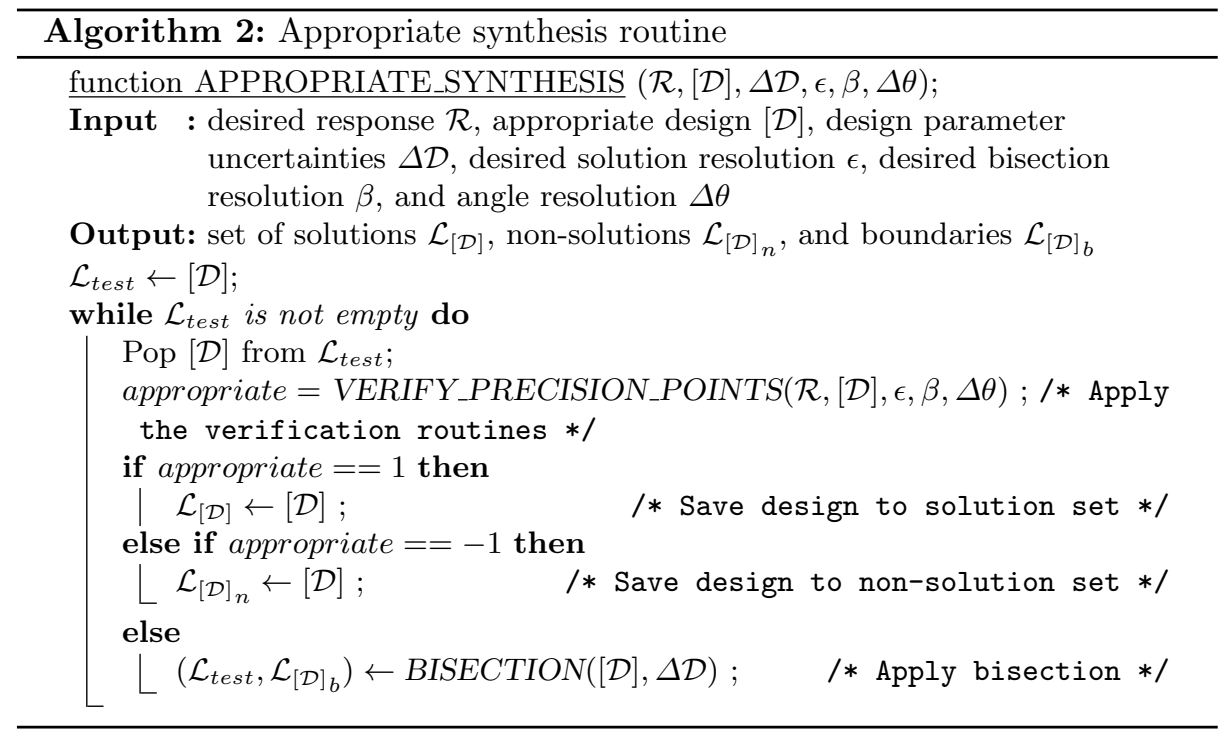

linkage which passes through each of the precision points even with uncertainties on the design parameters and input angle. For example, the appropriate design (9) is part of this solution set and satisfies the task.
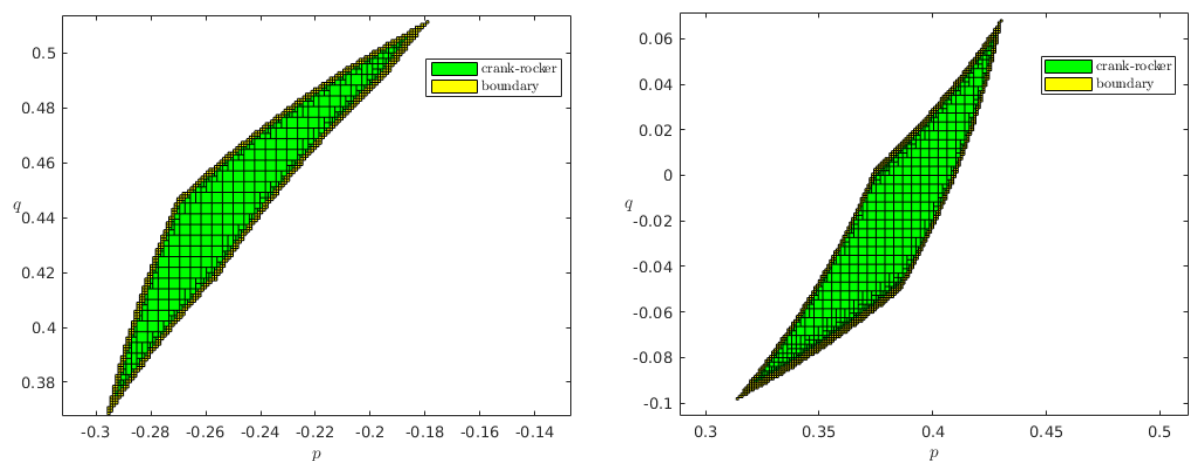

Fig. 3. The complete set of appropriate design solutions returned by the appropriate synthesis routine.

\section{Conclusions}

The appropriate design methodology applied to the crank-rocker linkage allows for understanding the effects of variability and uncertainties in the linkage. The 
coupler curve which corresponds to a linkage described by an appropriate design is modelled as the union of a set of coupler point boxes. An appropriate synthesis routine was developed to solve for the complete set of appropriate design solutions which corresponds to a given task. In this work, the task is modelled as a set of boxes (precision points) which the coupler point of the linkage must pass through. A linkage designer is free to select any design solutions from within the set of appropriate design solutions and certify that the resulting mechanism will perform as desired.

\section{References}

1. S. Bai and J. Angeles, Coupler-curve synthesis of four-bar linkages via a novel formulation, Mechanism and Machine Theory 94 (2015), 177-187.

2. R. R. Bulatović and S. R. Dordević, On the optimum synthesis of a four-bar linkage using differential evolution and method of variable controlled deviations, Mechanism and Machine Theory 44 (2009), no. 1, 235-246.

3. J.A. Cabrera, A. Simon, and M. Prado, Optimal synthesis of mechanisms with genetic algorithms, Mechanism and Machine Theory 37 (2002), no. 10, 1165-1177.

4. T. R. Chase and J. A. Mirth, Circuits and branches of single-degree-of-freedom planar linkages, Journal of Mechanical Design-Transactions of the ASME 115 (1993), no. 2, 223-230.

5. G. B. Chatterjee and A. K. Mallik, Mechanical error of a four-bar linkage coupler curve, Mechanism and Machine Theory 22 (1987), no. 1, 85-88.

6. V. Goulet, W. Li, H. Cheong, F. Iorio, and C.-G. Quimper, Four-bar linkage synthesis using non-convex optimization, pp. 618-635, Springer International Publishing, 2016.

7. K. H. Hunt, Kinematic geometry of mechanisms, Oxford engineering science series, Clarendon Press, 1978.

8. L. V. Kantorovich and G. E. Forsythe, Functional analysis and applied mathematics, NBS report, National Bureau of Standards, 1952.

9. R. Krawczyk, Newton-algorithmen zur bestimmung von nullstellen mit fehlerschranken, Computing 4 (1969), no. 3, 187-201.

10. J. McCarthy and G. Song Soh, Geometric design of linkages, Interdisciplinary Applied Mathematics, Springer, 2010.

11. J.-P. Merlet and D. Daney, Smart devices and machines for advanced manufacturing, ch. Appropriate Design of Parallel Manipulators, pp. 1-25, Springer London, London, 2008.

12. S. Roberts, On three-bar motion in plane space, Proceedings of the London Mathematical Society s1-7 (1875), no. 1, 14-23.

13. H. Schröcker, M. L. Husty, and J. McCarthy, Kinematic mapping based assembly mode evaluation of planar four-bar mechanisms, Journal of Mechanical Design 129 (2006), no. 9, 924-929.

14. C. W. Wampler, A. P. Morgan, and A. J. Sommese, Complete solution of the ninepoint path synthesis problem for four-bar linkages, Journal of Mechanical DesignTransactions of the ASME 114 (1992), no. 1, 153-159. 